\title{
A brain-actuated wheelchair: Asynchronous and non-invasive Brain-computer interfaces for continuous control of robots
}

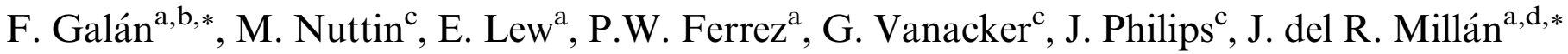 \\ ${ }^{a}$ IDIAP Research Institute, Centre du Parc., Av. des Prés-Beudin 20, CH-1920 Martigny, Switzerland \\ ${ }^{\mathrm{b}}$ University of Barcelona, Barcelona, Spain \\ ${ }^{\mathrm{c}}$ Department of Mechanical Engineering, Katholieke Universiteit Leuven, Leuven, Belgium \\ d Ecole Polytechnique Fédérale de Lausanne, Lausanne, Switzerland
}

Accepted 2 June 2008

\begin{abstract}
Objective: To assess the feasibility and robustness of an asynchronous and non-invasive EEG-based Brain-Computer Interface (BCI) for continuous mental control of a wheelchair.

Methods: In experiment 1 two subjects were asked to mentally drive both a real and a simulated wheelchair from a starting point to a goal along a pre-specified path. Here we only report experiments with the simulated wheelchair for which we have extensive data in a complex environment that allows a sound analysis. Each subject participated in five experimental sessions, each consisting of 10 trials. The time elapsed between two consecutive experimental sessions was variable (from $1 \mathrm{~h}$ to 2 months) to assess the system robustness over time. The pre-specified path was divided into seven stretches to assess the system robustness in different contexts. To further assess the performance of the brain-actuated wheelchair, subject 1 participated in a second experiment consisting of 10 trials where he was asked to drive the simulated wheelchair following 10 different complex and random paths never tried before.

Results: In experiment 1 the two subjects were able to reach 100\% (subject 1) and $80 \%$ (subject 2) of the final goals along the pre-specified trajectory in their best sessions. Different performances were obtained over time and path stretches, what indicates that performance is time and context dependent. In experiment 2, subject 1 was able to reach the final goal in $80 \%$ of the trials.

Conclusions: The results show that subjects can rapidly master our asynchronous EEG-based BCI to control a wheelchair. Also, they can autonomously operate the BCI over long periods of time without the need for adaptive algorithms externally tuned by a human operator to minimize the impact of EEG non-stationarities. This is possible because of two key components: first, the inclusion of a shared control system between the BCI system and the intelligent simulated wheelchair; second, the selection of stable user-specific EEG features that maximize the separability between the mental tasks.

Significance: These results show the feasibility of continuously controlling complex robotics devices using an asynchronous and noninvasive BCI.
\end{abstract}

(C) 2008 International Federation of Clinical Neurophysiology. Published by Elsevier Ireland Ltd. All rights reserved.

Keywords: Brain-computer interfaces; Electroencephalogram (EEG); Asynchronous protocol; Feature selection; Intelligent wheelchair; Shared control

\section{Introduction}

The possibility to act upon the surrounding environment without using our human nervous system's efferent path-

\footnotetext{
* Corresponding authors. Address: IDIAP Research Institute, Centre du Parc., Av. des Prés-Beudin 20, CH-1920 Martigny, Switzerland. Tel.: +41 277217770 ; fax: +41277217712.

E-mail addresses: ferran.galan@idiap.ch (F. Galán), jose.millan@ idiap.ch (J. del R. Millán).
}

ways enables a new interaction modality that can boost and speed up the human sensor-effector loop. In recent years, brain-computer interface (BCI) research is exploring many applications in different fields: communication, environmental control, robotics and mobility, and neuroprosthetics (Birbaumer et al., 1999; Obermaier et al., 2003; Bayliss, 2003; Millán, 2003; Nicolelis and Chapin, 2002; Millán et al., 2004; Carmena et al., 2003). Our work in 
the MAIA project ${ }^{1}$ aims at developing asynchronous and non-invasive BCI to control robots and wheelchairs (Millán et al., 2004; Lew et al., 2006). It means that users control such devices spontaneously and at their own paced, by learning to voluntarily control specific electroencephalogram (EEG) features measured from the scalp. To this end, users learn how to voluntarily modulate different oscillatory rhythms by the execution of different mental tasks (motor and cognitive). To facilitate this learning process, we rely upon machine learning techniques, both to find those subject-specific EEG features that maximize the separability between the patterns generated by executing the mental tasks (Galán et al., 2007), and to train classifiers that minimize the classification error rates of these subject-specific patterns (Millán et al., 2004). Finally, to assist the control task, different levels of intelligence are implemented in the device jointly with shared control techniques between the two interacting agents, the BCI system and the intelligent device (Philips et al., 2007; Vanacker et al., 2007).

One of the main challenges of a non-invasive BCI based on spontaneous brain activity is the non-stationary nature of the EEG signals. Shenoy and co-workers (Shenoy et al., 2006) describe two sources of non-stationarity, namely differences between samples extracted from calibration measurements (training data set) and samples extracted during the online operation of the BCI system (test data set), and changes in the user's brain processes during the online operation (e.g., due to fatigue, change of task involvement, etc). Such kinds of phenomena have motivated the BCI research groups to develop adaptive algorithms to deal with these shifts in the distributions of samples (Shenoy et al., 2006; Buttfield et al., 2006; Vidaurre et al., 2006; Millán et al., 2007). Unfortunately, current adaptive solutions have two main limitations. Firstly, they are based on supervised approaches requiring the correct output for every sample, and so the user cannot operate the BCI autonomously. Secondly, adaptation in the wrong moment (e.g., when the user is not properly executing the mental tasks because of fatigue, distraction, etc) will incorrectly change the feedback (the device's behavior) and will disrupt user's learning process. Given this scenario, two questions arise. Is it possible to find (rather) stable subject-specific EEG features to reduce the differences between samples extracted from calibration and online operation sessions? How shared control techniques can minimize the impact of changes in the user's EEG signals during the online operation?

In this paper we describe an asynchronous brain-actuated wheelchair that can be operated autonomously, and report results obtained by two subjects while continuously driving a simulated version of the wheelchair. Our brainactuated wheelchair exhibits two key components, namely

\footnotetext{
${ }^{1}$ MAIA - Mental Augmentation through Determination of Intended Action, http://www.maia-project.org.
}

the selection of stable user-specific EEG features that maximize the separability between the different mental tasks, and the implementation of a shared control system (Philips et al., 2007; Vanacker et al., 2007) between the BCI and the intelligent simulated wheelchair.

\section{Methods}

\subsection{EEG data acquisition and preprocessing}

EEG Data were recorded from two healthy subjects with a portable Biosemi acquisition system using 64 channels sampled at $512 \mathrm{~Hz}$ and high-pass filtered at $1 \mathrm{~Hz}$. Then, the signal was spatially filtered using a common average reference (CAR) before estimating the power spectral density (PSD) in the band $8-48 \mathrm{~Hz}$ with $2-\mathrm{Hz}$ resolution over the last $1 \mathrm{~s}$. The PSD was estimated every $62.5 \mathrm{~ms}$ (i.e., 16 times per second) using the Welch method with five overlapped $(25 \%)$ Hanning windows of $500 \mathrm{~ms}$. Thus, an EEG sample is a 1344-dimensional vector (64 channels $\times 21$ frequency components). Obviously, not all these 1344 features are used as control signals. Sections 2.2 and 2.3 describe the algorithms to estimate the relevance of the features for discriminating the mental commands and the procedure to select the most stable discriminant features that will be fed to the classifier embedded in the BCI. This classifier processes each of the EEG samples and the BCI combines eight consecutive responses to deliver a mental command every $0.5 \mathrm{~s}$.

\subsection{Calibration Sessions and Feature Extraction}

To extract stable discriminant EEG features (see Section 2.3.2) and build the statistical Gaussian classifier embedded in the BCI (see Section 2.3.3), both subjects participated in 20 calibration sessions recorded in the same day as the test driving session 1 . The calibration sessions were recorded during the morning and the test driving session 1 during the afternoon. As in the driving sessions, the subjects sat on a chair looking at a fixation point in the center of a monitor. The display was also the same, the simulated wheelchair being in a first person view (see Fig. 1, left). The subjects were instructed to execute the three mental tasks (left hand imagination movement, rest, and words association), ${ }^{2}$ tasks utilized as mental commands to operate the wheelchair, in a self-paced way. The mental task to be executed was selected by the operator in order to counterbalance the order, while the subjects decided when they started to execute the mental task. Each calibration session was integrated by six trials each, two trials of each class. The duration of each trial was $7 \mathrm{~s}$ but only the last $6 \mathrm{~s}$ were utilized in the analysis to avoid preparation periods where

\footnotetext{
${ }^{2}$ The mental tasks consisted in imagining repetitive self-paced movements of the left hand, getting relaxed centering attention on the fixation point placed on the center of the monitor, and searching words starting with the same letter.
} 

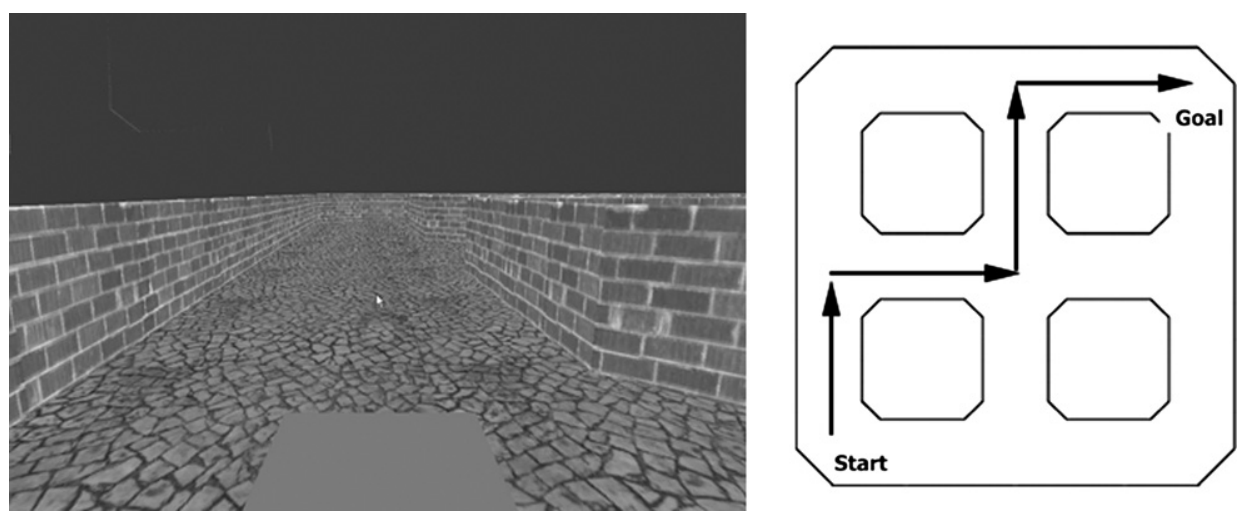

Fig. 1. (Left) Monitor display in a first person view from the Start. The white cursor at the center is the fixation point. The rectangle at the bottom is the simulated wheelchair. (Right) Top view of the simulated world and the pre-specified path.

the subjects were not yet engaged in the execution of the mental task. A trial started when subjects informed the operator they were executing the requested mental task. In these sessions the subjects did not receive any feedback, so the monitor display was static, i.e., the simulated wheelchair did not move.

The data from the 20 calibration sessions were grouped in four blocks (B1, B2, B3 and B4) of five consecutive sessions. Taking into account the recordings timing, there were different configurations of training and testing sets (train - test): B1 - B2, B1 - B3, B1 - B4, B2-B3, $\mathrm{B} 2-\mathrm{B} 4, \mathrm{~B} 3-\mathrm{B} 4,(\mathrm{~B} 1+\mathrm{B} 2)-\mathrm{B} 3,(\mathrm{~B} 1+\mathrm{B} 2)-\mathrm{B} 4$, and $(\mathrm{B} 1+\mathrm{B} 2+\mathrm{B} 3)-\mathrm{B} 4$. Feature selection was done in a sequential way, where we first picked stable frequency components and then chose the best electrodes. To assess the stability of the frequency components we applied 21 canonical variates analysis (CVA), one per frequency component, on the training set of each configuration. For each canonical space we ranked the electrodes according to their contribution to this space (see Section 2.3.2). Then, we built up to 15 linear discriminant (LDA) classifiers ${ }^{3}$, each using those electrodes that contributed more than $c \%$, with $c \in\{1.0,2.0, \ldots, 15.0\}$. We used the stability of the classifier accuracy over the different configurations to select the frequency components. In particular, we selected those frequencies that performed systematically among the top 5 . Afterwards, for each selected frequency, we took the configuration of electrodes (out of the 15 possible ones) that yielded the highest classification accuracy on the configuration $(\mathrm{B} 1+\mathrm{B} 2+\mathrm{B} 3)-\mathrm{B} 4$. Finally, we tested the different combinations of selected frequencies (with their associated electrodes) on the configuration (B1 + B2 + B3) - B4 and chose the best one. At the end of this sequential process the selected frequencies were $12 \mathrm{~Hz}$ for subject 1 and $\{10,12,14\} \mathrm{Hz}$ for subject 2 . We then built the statistical Gaussian classifier (see Section 2.3.3) for each subject using

\footnotetext{
${ }^{3}$ The reasons for using a LDA classifier for feature extraction rather than the final Gaussian classifier are the simplicity and speed of training of the former. Furthermore, LDA is a special case of our Gaussian classifier.
}

their individual selected features from all the data of the calibration sessions. Table 1 reports the LDA classifier accuracies on the configuration $(\mathrm{B} 1+\mathrm{B} 2+\mathrm{B} 3)-\mathrm{B} 4$ using the selected features for each subject. Accuracies are not very high, what is normal for a first session without feedback, but still well above random level (33.3\% for a 3-class classification problem).

Fig. 2 depicts the electrodes contribution, for each selected frequency component for each subject, and the associated scalp distribution of the averaged logarithmic transform of the PSD $\left(\log \left(\mathrm{PSD}_{\mathrm{e}}\right)\right)$ for each mental task. The $\log \left(\mathrm{PSD}_{\mathrm{e}}\right)$ scalp distributions show that the differences between the mental tasks, localized in those electrodes with higher $D_{\mathrm{e}}$ values (see Section 2.3.2) are bigger for subject 2 . This is in agreement with the train classification accuracies, $59 \%$ and $85 \%$, for subjects 1 and 2 , respectively.

\subsection{System description}

The system is integrated by two entities, the intelligent wheelchair and the BCI system. Environmental information from the wheelchair's sensors feeds a contextual filter that builds a probability distribution $P_{\text {Env }}(C)$ over the possible user's mental steering commands, $C=\{$ Left, Right, Forward $\}$. The BCI system estimates the probabilities $P_{\mathrm{EEG}}(C)$ of the different mental commands from the EEG signals. Both streams of information are combined to produce a filtered estimate of the user's intent $P(C)=P_{\mathrm{EEG}}(C) \cdot P_{\mathrm{Env}}(C)$. The shared control system also uses the environmental information from the wheelchair's

Table 1

LDA train-test classification accuracies on the configuration $(\mathrm{B} 1+\mathrm{B} 2+\mathrm{B} 3)-\mathrm{B} 4$ using the selected features for each subject

\begin{tabular}{lll}
\hline Subject & Train $(\%)$ & Test $(\%)$ \\
\hline 1 & 59.0 & 54.7 \\
2 & 85.0 & 61.2 \\
\hline
\end{tabular}




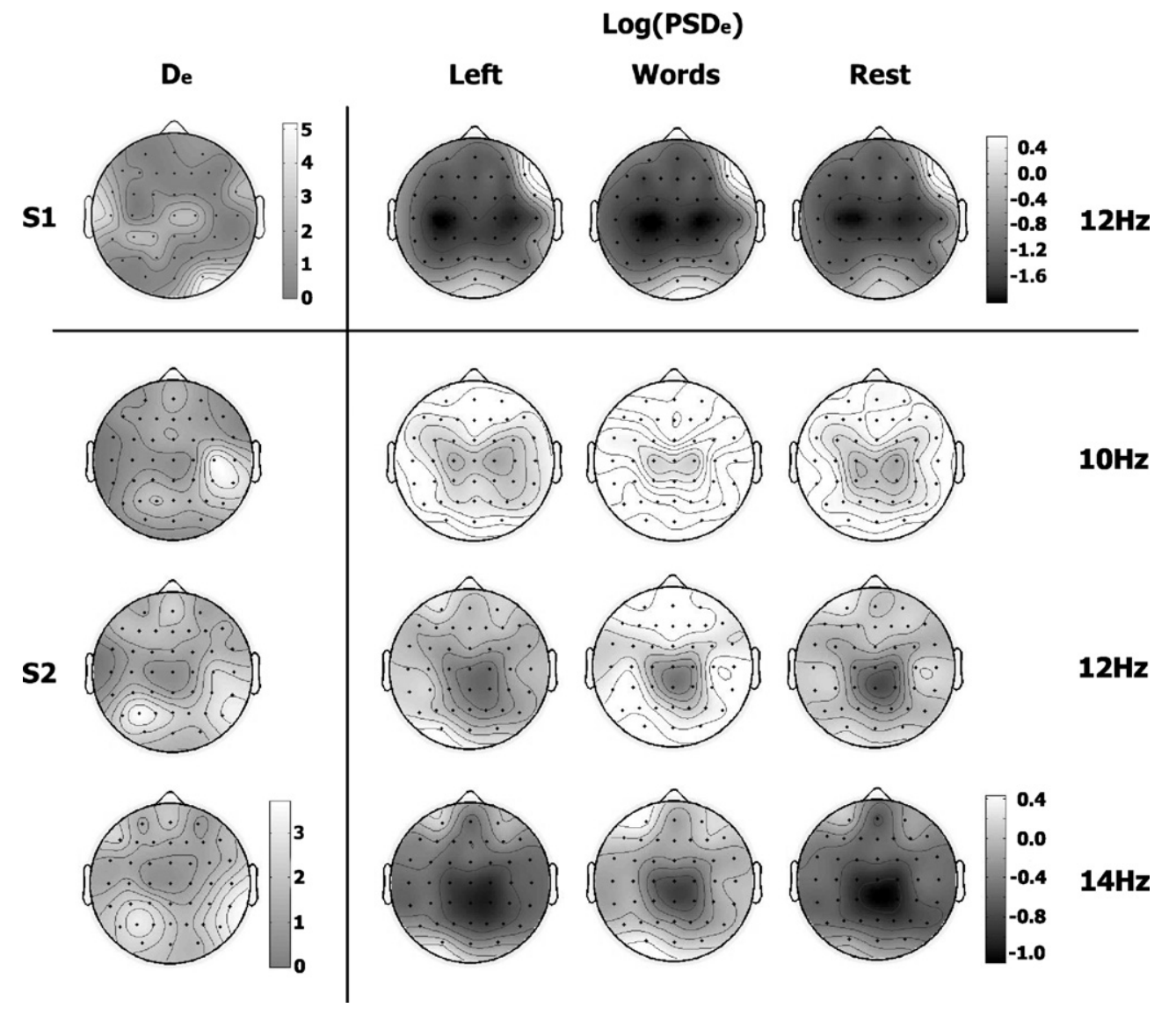

Fig. 2. Electrode discrimination index values $D_{\mathrm{e}}$ (see Section 2.3.2) for the selected frequencies for each subject, and the associated scalp distribution of the averaged logarithmic transform of the power spectral density, $\log \left(\mathrm{PSD}_{\mathrm{e}}\right)$, for each class. For subject $1, D_{\mathrm{e}}$ is higher at left temporal, central and right occipital areas. For subject 2, at $10 \mathrm{~Hz}$ it is higher at right centro-parietal areas, and at 12 and $14 \mathrm{~Hz}$ it is higher at bilateral parietal areas. These areas correspond with those where the differences between the averaged $\log \left(\mathrm{PSD}_{\mathrm{e}}\right)$ patterns associated to each mental task is the biggest.

sensors to map these high-level commands into appropriate motor commands, translational and rotational velocities, that generates a smooth and safe driving behavior. This is achieved by constantly adapting the level of assistance provided to the user to negotiate obstacles. Thus, the intelligent wheelchair, via shared control, will significantly help when the subject's performance (BCI accuracy) is low whereas it will decrease its role when the BCI accuracy is higher. In other words, the intelligent wheelchair will take over control to avoid obstacles, if subjects cannot deliver the proper mental commands to stay at a safe distance from obstacles and will not activate any assisting behavior in case subjects can safely drive the wheelchair. Obstacle avoidance is the only assisting behavior used in the experiments. Fig. 3 depicts a schematic representation of the shared control architecture of the brain-actuated wheelchair. See Philips et al. (2007) and Vanacker et al. (2007) for a detailed description. As for the BCI, it has two components: a feature extractor and a Gaussian classifier. The former selects the most relevant features of the EEG signals based on canonical variates analysis (Galán et al., 2007). Based on these features, the Gaussian classifier estimates the probability distributions of the three mental commands (Millán et al., 2004).

\subsubsection{Context-based filter}

Context estimation is done by defining a general, a priori-known user intention (smooth and efficient forward navigation through the environment) on the one hand and a constant automatic estimation of the environmental situation on the other hand. The situations are modelled as the number and location of openings: wide, open spaces to which the user might safely navigate. The principle is as follows: suppose the wheelchair is approaching a crossroad, as depicted in Fig. 4. The laser scanner in front of the wheelchair scans $180^{\circ}$ and senses the distance to the environment for every degree. The algorithm then searches for regions with consecutive scans for which the distance is larger than a certain threshold T. This results in a number of regions that qualify as candidates for an opening. Next, for each of the resulting regions, the width of the opening $O$ is calculated: $O=\sqrt{s_{1}^{2}+s_{2}^{2}-2 s_{1} s_{2} \cos \left(t_{2}-t_{1}\right)}$. This length is then compared to the physical dimensions of the wheelchair. If the length $O$ exceeds the wheelchair width augmented with a safety margin, the corresponding region is accepted as an opening. Its orientation with respect to the current wheelchair position is then $\frac{\pi}{2}-\frac{t_{2}-t_{1}}{2}$.

Each opening then represents a general direction in which the user might opt to continue his navigation. With 


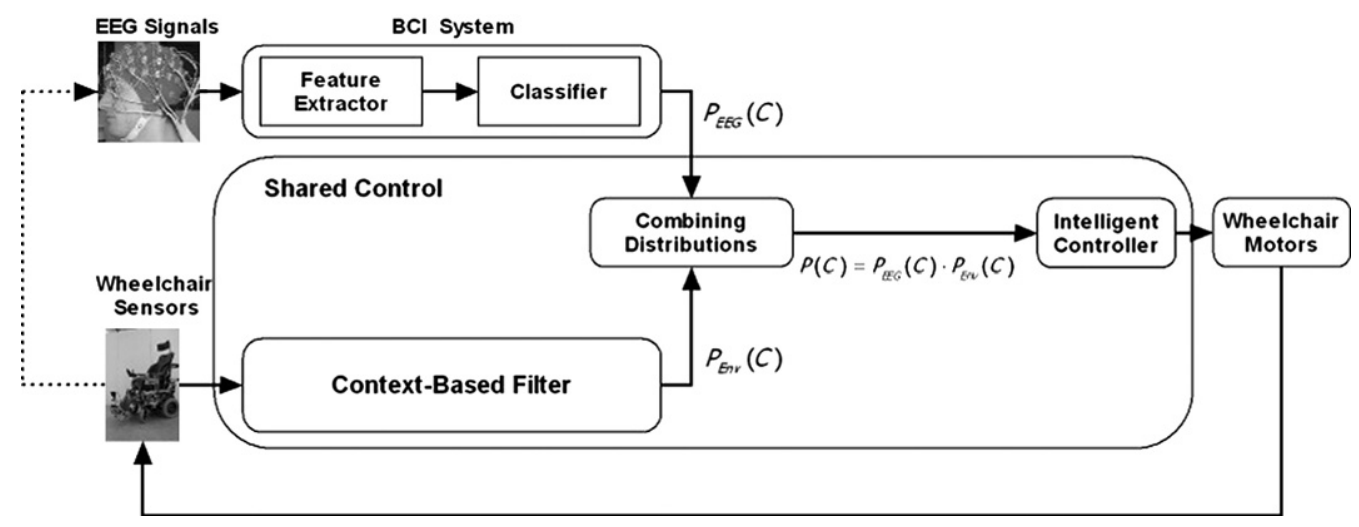

Fig. 3. Architecture of the brain-actuated wheelchair.

this knowledge about the current situation, a probability distribution concerning the possible local user actions is built. Note that inferring these probabilities requires the knowledge of the global intention of the human. In this case, it is supposed that the user wishes to navigate safely and efficiently through the environment without halting or going backwards. In other cases, a user might also wish to stop at certain locations, or dock at particular places. When the directions in which the robot can travel are orthogonal, as in Fig. 4, it is possible to summarize the environmental belief in four quadrants, as depicted in Fig. 5. The figure shows how the regions West and North are deemed probable navigation directions, as extracted from the environment (see Fig. 4). The regions East and South are improbable (as the scanner sees a wall on the right hand, and going backwards is also not probable given

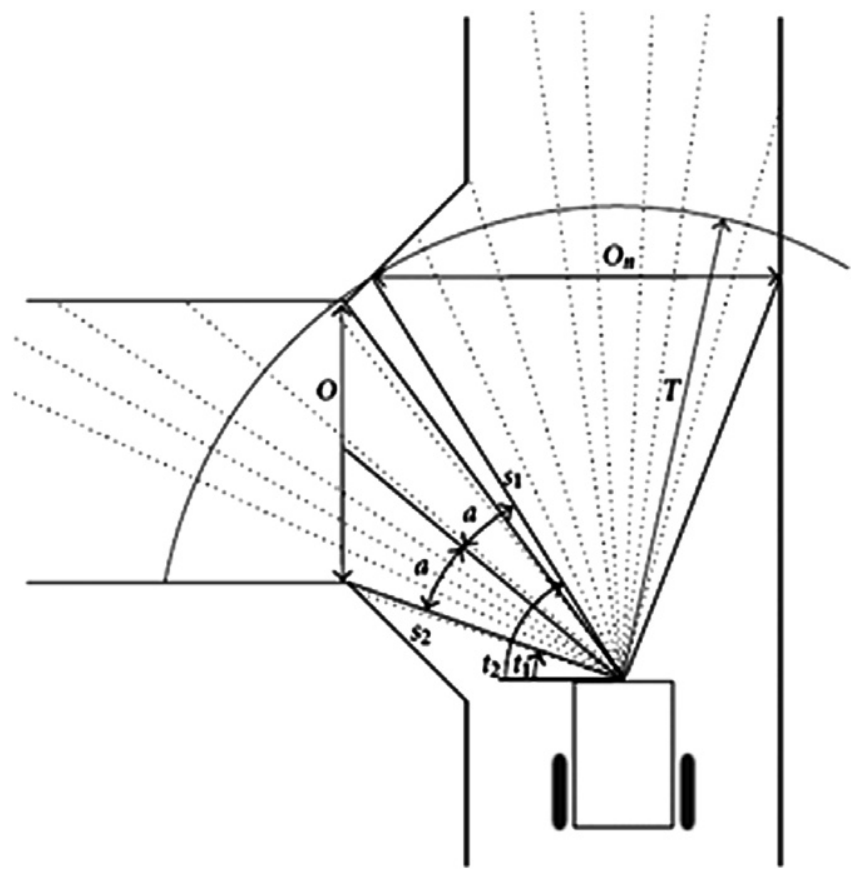

Fig. 4. Principle of context estimator. With a laser range scanner, a set of regions that provide safe manoeuvrable openings in the environment is detected. The figure shows how the region to the left and the one in front of the wheelchair are detected as openings. the intention of smooth forward navigation). If the wheelchair is oriented North, the controller attaches a probability of 0.5 to Forward and Left. $P_{\text {Env }}$ (Right) is set to zero, because rotating to the right would make the robot turn towards an obstacle (the wall). The possibility of turning into the corridor to the left is reflected in $P_{\text {Env }}($ Left $)=0.5$. If the wheelchair is oriented $45^{\circ}$ North-West, $P_{\text {Env }}($ Forward) becomes zero, while the possible commands now are Left and Right, with equal probability, reflecting the belief that one of the orthogonal directions North or West should be chosen. When the wheelchair is turning further towards West, Forward becomes possible again, and $P_{\text {Env }}($ Right $)$ stays constant while $P_{\text {Env }}($ Left $)$ diminishes completely. At the boundary between the probable directions and those that are improbable, the controller attaches

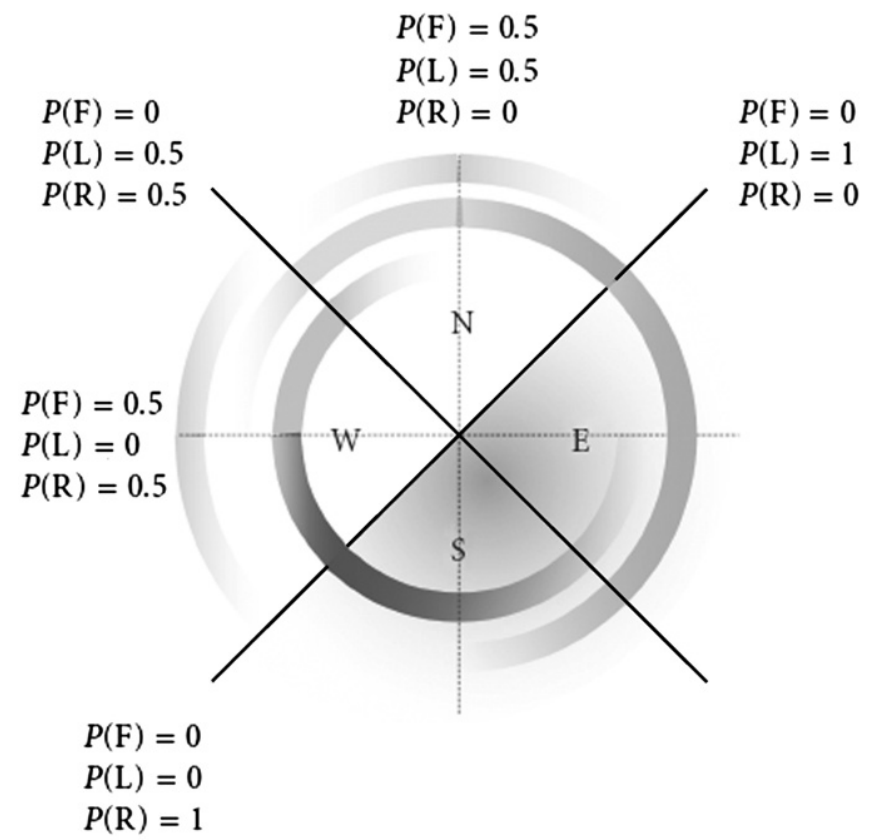

Fig. 5. Environmental belief over the four quadrants in function of the wheelchair orientation in Fig. 4. The inner circle shows the probability of a Right command $\mathrm{P}(\mathrm{R})$, the middle circle shows the probability of a Left command $\mathrm{P}(\mathrm{L})$, and the outer circle the probability of a Forward command $\mathrm{P}(\mathrm{F})$. The figure also shows the resulting probabilities of the three steering command along each direction. 
a maximum belief to those commands that would keep the wheelchair in the half plane of high probability. Between the above-described orientations, the probabilities are interpolated linearly. This is depicted in Fig. 5 as the linearly changing transparency of the respective circle. See Vanacker et al. (2007) for a detailed description.

\subsubsection{Feature extractor}

Our approach is based on a mutual learning process where the user and the BCI are coupled together and adapt to each other. To facilitate and accelerate this process, it is necessary to select the relevant EEG features that best discriminate among the mental tasks executed by the user. The feature selection process is based on Canonical Variates Analysis (CVA) (Krzanowski, 1988), also known as Multiple Discriminant Analysis (Duda et al., 2001), which provides a canonical solution for multi-class problems. In our case, CVA extracts Canonical Discriminant Spatial Patterns (CDSPs) whose directions maximize the differences in mean spectral power between a given number of classes.

Let $\mathbf{S}_{k}=\left(\mathbf{s}_{k 1}, \ldots, \mathbf{s}_{k n_{k}}\right)^{\prime}$ be the $n_{k} \times c$ matrix with the estimated spectral power of a frequency band for class $k=1, \ldots, l$, where $n_{k}$ is the number of samples and $c$ is the number of channels. Now, given $\mathbf{S}=\left(\mathbf{S}_{1}^{\prime}, \ldots, \mathbf{S}_{l}^{\prime}\right)^{\prime}$, the $l-1$ CDSPs of $\mathbf{S}$ are the eigenvectors $\mathbf{A}$ of $\mathbf{W}^{-1} \mathbf{B}$ whose eigenvalues $\lambda_{u},(u=1, \ldots, l-1)$ are larger than 0 . Note that the direction of the eigenvectors $\mathbf{A}$ maximizes the quotient between the between-classes dispersion matrix $\mathbf{B}$ and the pooled within-classes dispersion matrix $\mathbf{W}$. Thus, the CDSPs are obtained by projecting $\mathbf{X}=\mathbf{S A}$.

Once the CDSPs are computed, it is useful to know how the original channels are contributing to the separability among the classes. To measure this contribution we compute a Discrimination index for each channel from the structure matrix - the pooled correlation matrix between the original channels in $\mathbf{S}$ and the CDSP $\mathbf{X}$. Given the $c \times(l-1)$ structure matrix $\mathbf{T}$, where $\mathbf{T}=\sum_{k=1}^{l} \mathbf{T}_{k}, e=$ $1, \ldots, c$, and the normalized eigenvalues $\gamma_{u}=\lambda_{u} / \sum_{u=1}^{l-1} \lambda_{u}$, the proposed discrimination index is computed as $D_{e}=\left(\sum_{u=1}^{l-1} \gamma_{u} t_{e u}^{2} / \sum_{e=1}^{c} \sum_{u=1}^{l-1} \gamma_{u} t_{e u}^{2}\right) \times 100$. See Galán et al. (2007) for more details.

\subsubsection{Classifier}

The classifier utilized is a statistical Gaussian classifier (see Millán et al., 2004 for more details). The output of this statistical classifier is an estimation of the posterior class probability distribution for a sample; i.e., the probability that a given single trial belongs to each mental task (or class). Each class is represented by a number of Gaussian prototypes, typically less than four. That is, it is assumed that the class-conditional probability function of class $k$ is a superposition of $N_{k}$ Gaussian prototypes. It is also assumed that all classes have equal prior probability. All classes have the same number of prototypes $N_{p}$, and for each class each prototype has equal weight, $1 / N_{k}$. Then, dropping constant terms, the activity of the $i^{\text {th }}$ prototype of class $k$ for a given sample $\mathbf{x}$ is the value of the Gaussian with center $\mu_{k}^{i}$ and covariance matrix $\Sigma_{k}^{i}$. From this we calculate the posterior probability $y_{k}$ of the class $k$, which is the sum of activities of all the prototypes of class $k$ divided by the sum of the activities of all the prototypes of all the classes. The classifier output for input vector $\mathbf{x}$ is then the class with the highest probability. In order to smooth this output, we average the class-conditioned probabilities of the last eight consecutive input vectors $\mathbf{x}$. Thus, the BCI responds every $0.5 \mathrm{~s}$. Usually each prototype of each class would have an individual covariance matrix $\Sigma_{k}^{i}$, but to reduce the number of parameters the model has a single diagonal covariance matrix common to all the prototypes of the same class. During offline training of the classifier, the prototype centers are initialized by any clustering algorithm or generative approach. This initial estimate is then improved by stochastic gradient descent to minimize the mean square error $E=\frac{1}{2} \sum_{k}\left(y_{k}-t_{k}\right)^{2}$, where $\mathbf{t}$ is the target vector in the form 1-of-C; that is, if the second of the three classes was the desired output, the target vector is $(0,1,0)$. The covariance matrices are computed individually and are then averaged over the prototypes of each class to give $\Sigma_{k}$.

\subsection{Experimental tasks}

\subsubsection{Task 1}

Both subjects sat in a chair looking at a fixation point placed in the center of a monitor. The monitor displayed a simulated wheelchair in a first person view moving in a simulated world. The subjects were asked to mentally drive the simulated wheelchair from a starting point to a goal following a pre-specified path by executing three different mental tasks (left hand imagination movement to turn Left, rest to go Forward, and words association to turn Right). Fig. 1 depicts the monitor display and the pre-specified path. Every subject participated in five experimental sessions, each consisting of 10 trials. The time elapsed between two consecutive experimental sessions was variable to assess the system robustness over time: 1 day between sessions 1 and 2, 2 months between sessions 2 and 3, $1 \mathrm{~h}$ between sessions 3 and 4, and finally 1 day between sessions 4 and 5.

\subsubsection{Task 2}

To further assess the performance of the brain-actuated wheelchair, Subject 1 participated in a second experiment four months later. He performed 10 trials in the same simulated environment where he was asked to drive the simulated wheelchair following 10 different complex and random paths never tried before. Fig. 6 depicts the 10 complex and random paths. Subject 2 did not participate in this task because she was not available.

\subsection{Analysis}

The system's robustness was assessed in task 1 on three criteria, namely the percentage of goals reached, the BCI 

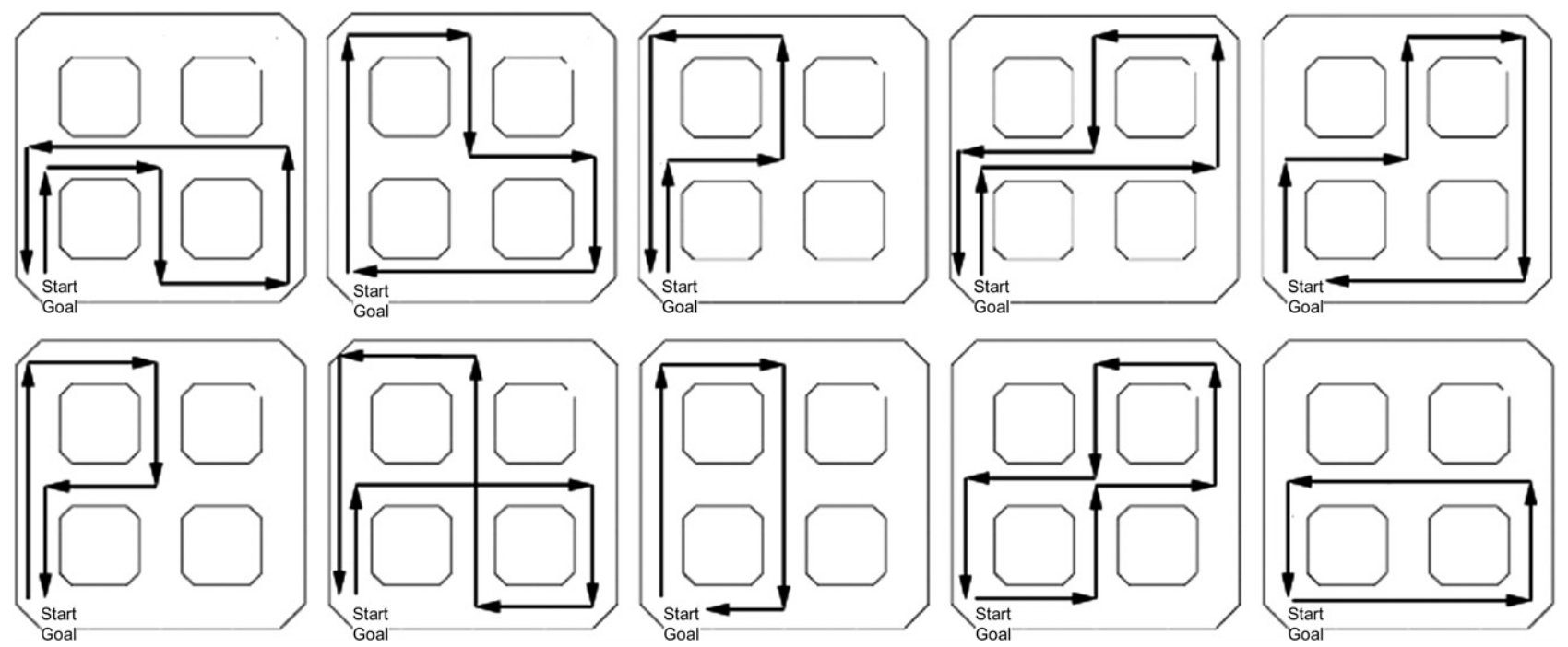

Fig. 6. Top view of the random paths in Task 2. Trial 1 placed in upper row, first column. Trial 10 placed in second row, last column.

classification accuracy, and the shared control accuracy (the actual mental commands sent to the wheelchair after combining the probability distributions from the BCI and contextual filter). The three criteria were analyzed over time ( 5 sessions) and context. For the contextual analysis, the path was split into seven stretches. Thus, the system's performance was measured over the final goal (complete path) and subgoals (path stretches). Additionally, we compared the performance of the two subjects to that of a random BCI to further assess their level of mental control. In this case, we use the percentage of goals reached by a random BCI as a reference.

The analysis of the accuracies of the BCI and shared control has a main limitation since it requires to know the subject's intent. It is true, however, that in the experiments subjects had to verbally inform the operator whenever they switched mental task so that the latter could label the data. Unfortunately, this approach is far from optimal. Indeed, providing this information interferes with, and so hampers, the driving task. As a consequence, the subject may deliver wrong or delayed mental commands leading to poor trajectories that the subject needs to correct by rapidly switching between mental commands - and the subject does not have time to inform the operator of all those switches and their exact timing. It follows that using the subject's stated intent for labelling data yields a pessimistic and/or wrong estimate of the accuracies of the $\mathrm{BCI}$ and the shared control. For this reason the accuracies were estimated in a different way. Each path stretch was labelled with the command that makes the wheelchair reach the next subgoal. Only those samples where the subject's stated intent corresponds to the stretch label were utilized to compute the accuracies. Fig. 7 shows the seven labelled stretches.

To avoid the limitations described before, in task 2 the subject drove the wheelchair without informing the operator about the mental command he was executing. In this way, the subject could drive the simulated wheelchair in real conditions that allow a better assessment of the brain-actuated wheelchair. In this case only the behavioral performance (percentage of goals reached) was assessed.

An issue to be ruled out in any BCI system is the use of eye movements or muscular activity components embedded in the EEG as control signals. In the experiments described in this paper this issue was not assessed directly, but it was in the posterior experiments with the real wheelchair where the two subjects utilized the same statistical Gaussian classifier as here. In these experiments we monitored eye movements and muscular activity by means of bipolar electrooculogram (EOG) using surface electrodes placed

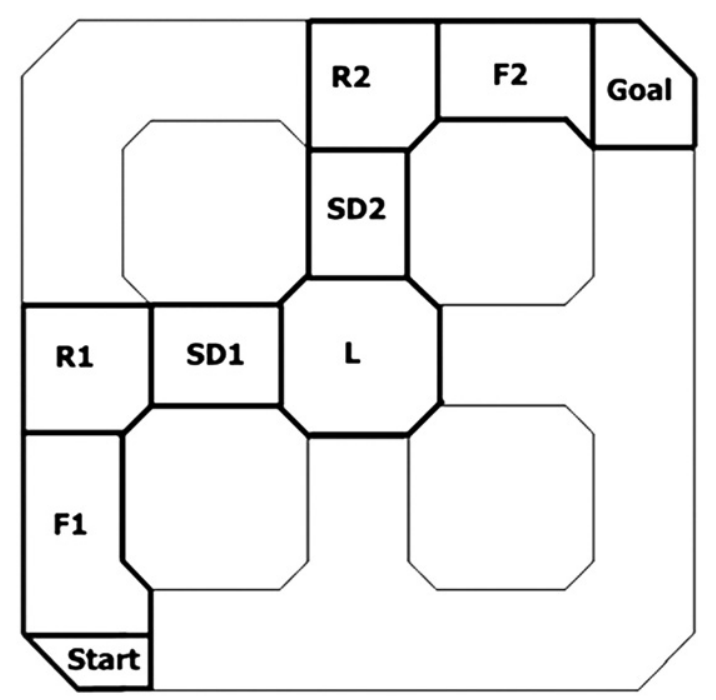

Fig. 7. Top view of the world and the path stretches. Stretches F1 and F2 were labelled as Forward, R1 and R2 labelled as Right, L labelled as Left, and SD1 and SD2 labelled as strategy dependent. The subjects can go through SD1 by means of two strategies, either executing Forward or executing Right followed by Left. Through SD2, subjects can execute either Forward or Left followed by Right. 
below and laterally to the left eye, and by bipolar electromyogram (EMG) using two surface electrodes placed on the forearm muscle Extensor Digitorum. The analysis of EOG and EMG activity showed that eye movements were equally distributed among the classes and that there was no significant muscular activity. Thus, we can conclude that subjects did not use any EOG and/or EMG feature as control signals. Also, the fact that the selected band frequency is $10-14 \mathrm{~Hz}$ makes it very improbable to have EOG/EMG artifacts. Furthermore, in the experiments reported in this article, we did not observe any overt movement of the subjects' left hand.

\section{Results}

\subsection{Task 1}

\subsubsection{Global performance}

Fig. 8 depicts the percentage of final goals reached over the five sessions for the two experimental subjects. Subject 1 reached more final goals in all the sessions. For both subjects, sessions 1 and 3 are the sessions with less reached final goals $(40 \%$ and $10 \%$ in session $1,50 \%$ and $40 \%$ in session 3). Note that between session 2 and session 3 passed 2 months, so sessions 1 and 3 can be considered as sessions where the subjects learn (session 1) and re-learn (session 3 ) how to interact with the system and its dynamics. If these sessions were not considered, the average percentage of reached final goals are $86.7 \%$ and $66.7 \%$ for subjects 1 and 2 , respectively. Regarding the maximum performances, subject 1 reached the final goal in $100 \%$ of the trials in session 4 , and subject 2 reached the final goal in $80 \%$ of the trials in session 2. It is worth noting that even in the first session where the subjects had the lowest performance ( $40 \%$ and $10 \%$ of reached goals), they significantly outperformed the random BCI that only reached the goal along the pre-specified path in $1 \%$ of the cases. This figure was obtained by running 100 trials.

Table 2 displays the percentage of reached local goals, the average BCI classification accuracy and the shared con-

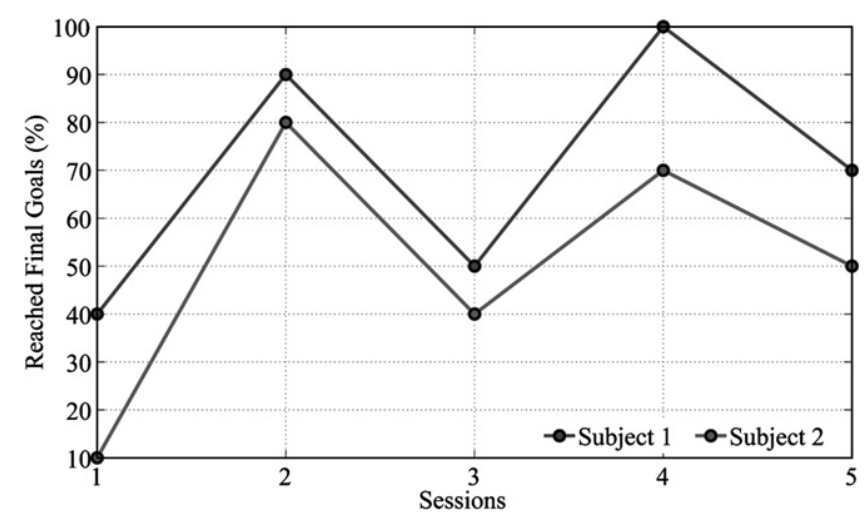

Fig. 8. Percentage of reached final goals over sessions. The time elapsed between sessions was: 1 day between sessions 1 and 2, 2 months between sessions 2 and $3,1 \mathrm{~h}$ between sessions 3 and 4, and 1 day between sessions 4 and 5 . trol accuracy on each session over the seven path stretches (local goals) for the two subjects, and the percentage of reached goals for the random BCI. This table makes clear the reasons why subjects couldn't reach the final goal they failed sometimes to turn Left at the stretch L and/or to turn Right at the stretches R1 and R2. Interestingly, in these three stretches shared control performed generally worse than the BCI, what could indicate that subjects tried to deliver mental commands that the shared control system considers impossible to execute. On the contrary, shared control significantly improved the performance of BCI at stretches F1, SD1, SD2 and F2, where the wheelchair was supposed to go straight. The average difference over these stretches is $35 \%$ for subject $1(24 \%$ BCI vs. $59 \%$ shared control) and $20 \%$ for subject $2(34 \%$ BCI vs. $55 \%$ shared control). These 'poor' accuracies of the BCI and shared control indicate that to drive the wheelchair straight subjects cannot simply deliver the mental command Forward, but needed to steer Left and Right. Furthermore, shared control helped to generate smoother trajectories, especially in the vicinity of walls.

Subject 1 failed to reach the final goal in session 1 because he could not turn Left at stretch L in 30\% of the cases and, afterwards, he failed to turn Right in $40 \%$ of the cases in which he successfully arrived stretch R2. In this session, subject 1 always correctly performed the optimal action for all other stretches he went through. As mentioned before, at these 'hard' stretches, L and R2, shared control degraded the BCI performance $(50 \%$ vs. $62 \%$ in L and $47 \%$ vs. $53 \%$ in R2). Regarding session 3, subject 1 failed to reach the final goal because he could not turn Left at stretch $\mathrm{L}$ in $50 \%$ of the cases. This was due to a low BCI accuracy $(42 \%)$ and a lower shared control accuracy $(37 \%)$. Finally, in sessions 2, 4 and 5 subject 1 reached the final goal in $70 \%$ (or more) of the trials and each local goal in more than $88 \%$ of the trails.

Subject 2 failed to reach the final goal in session 1 because he could not turn Right at stretch R1 in $90 \%$ of the cases. This was due to a very low BCI and shared control accuracy (29\%). In sessions 3 and 5, the poor final performance was due to failures in turning Left at stretch $\mathrm{L}-$ accuracies of $50 \%$ and $40 \%$, respectively. Similarly to subject 1 , also in these two sessions shared control degraded the BCI performance although less severely ( $38 \%$ vs. $37 \%$ in session 3, $48 \%$ vs. $39 \%$ in session 5). Finally, in sessions 2 and 4 subject 2 reached the final goal in $70 \%$ (or more) of the trials and each local goal in more than $80 \%$ of the trials.

Regarding the random BCI, it reached the final goal a mere $1 \%$ of the trials because it was able to turn Right at stretch R1 and to turn Left at stretch L only $16 \%$ and $6 \%$ of the trials, respectively, percentages significant lower than those achieved by subjects 1 and 2 .

\subsubsection{System performance in single trials}

Here we analyze the performance of the brain-actuated wheelchair in a few single trials to illustrate emergent 
Table 2

Percentage of local goals reached (subgoals), average BCI classification accuracy and average shared control accuracy over the 7 path stretches

\begin{tabular}{|c|c|c|c|c|c|c|c|c|c|}
\hline \multirow[t]{2}{*}{ Subject } & \multirow[t]{2}{*}{ Criterion } & \multirow[t]{2}{*}{ Session } & \multicolumn{7}{|c|}{ Path stretch } \\
\hline & & & F1 & $\mathrm{R} 1$ & SD1 & $\mathrm{L}$ & SD2 & R2 & $\mathrm{F} 2$ \\
\hline \multirow[t]{10}{*}{1} & Subgoals (\%) & 1 & 100 & 100 & 100 & 70 & 100 & 57 & 100 \\
\hline & & 2 & 100 & 100 & 100 & 90 & 100 & 100 & 100 \\
\hline & & 3 & 100 & 100 & 100 & 50 & 100 & 100 & 100 \\
\hline & & 4 & 100 & 100 & 100 & 100 & 100 & 100 & 100 \\
\hline & & 5 & 100 & 90 & 100 & 89 & 100 & 88 & 100 \\
\hline & $\mathrm{BCI} /$ shared control accuracy $(\%)$ & 1 & $18 / 45$ & $73 / 62$ & $20 / 40$ & $62 / 50$ & $18 / 33$ & $53 / 47$ & $23 / 67$ \\
\hline & & 2 & $22 / 52$ & $73 / 70$ & $26 / 53$ & $57 / 55$ & $20 / 58$ & $68 / 67$ & $19 / 58$ \\
\hline & & 3 & $34 / 62$ & $70 / 59$ & $22 / 46$ & $42 / 37$ & $15 / 78$ & $69 / 63$ & $29 / 85$ \\
\hline & & 4 & $28 / 55$ & $70 / 63$ & $22 / 66$ & $54 / 51$ & $16 / 57$ & $69 / 64$ & $25 / 68$ \\
\hline & & 5 & $33 / 62$ & $56 / 51$ & $29 / 62$ & $53 / 52$ & $29 / 63$ & $56 / 47$ & $30 / 75$ \\
\hline \multirow[t]{10}{*}{2} & Subgoals $(\%)$ & 1 & 100 & 10 & 100 & 100 & 100 & 100 & 100 \\
\hline & & 2 & 100 & 100 & 100 & 90 & 100 & 89 & 100 \\
\hline & & 3 & 100 & 100 & 100 & 40 & 100 & 100 & 100 \\
\hline & & 4 & 100 & 80 & 100 & 88 & 100 & 100 & 100 \\
\hline & & 5 & 100 & 100 & 100 & 50 & 100 & 100 & 100 \\
\hline & $\mathrm{BCI} /$ shared control accuracy $(\%)$ & 1 & $40 / 61$ & $29 / 29$ & $17 / 42$ & $89 / 89$ & $25 / 83$ & $61 / 68$ & $36 / 50$ \\
\hline & & 2 & $33 / 41$ & $71 / 68$ & $40 / 62$ & $57 / 59$ & $26 / 48$ & $66 / 65$ & $35 / 61$ \\
\hline & & 3 & $40 / 55$ & $77 / 75$ & $40 / 57$ & $38 / 37$ & $26 / 56$ & $73 / 67$ & $48 / 70$ \\
\hline & & 4 & $38 / 46$ & $62 / 63$ & $46 / 62$ & $49 / 53$ & $38 / 48$ & $77 / 77$ & $35 / 61$ \\
\hline & & 5 & $31 / 42$ & $65 / 63$ & $27 / 43$ & $48 / 39$ & $27 / 43$ & $77 / 74$ & $24 / 54$ \\
\hline Random & Subgoals $(\%)$ & - & 100 & 16 & 100 & 6 & 100 & 100 & 100 \\
\hline
\end{tabular}

behaviors originated by the interaction of the BCI system and the shared control system in particular contexts. The experimental results show that subjects cannot execute a given mental task with the same level of proficiency all across the trajectories and over time. But, is this the only reason of the inter-trial differences in BCI classification accuracy for the same path stretch? We have observed that the interaction of the BCI system and the shared control system in a particular context also plays a significant role. We have already mentioned in the previous section that, for some stretches, shared control degraded the performance of the BCI, what could indicate that subjects tried to deliver mental commands that the shared control system considers impossible to execute. Here we take a closer look at this situation.

Table 3 shows the performance for subject 1 in session 4 for trials 2 and 8 at two stretches, R1 and R2, requiring the same command. Subject 1 always succeeded in making the wheelchair turn Right. However, the BCI and shared control performances were rather different. Thus, we can see that whenever the BCI accuracy is sufficiently high $(92 \%$ in trial 2 stretch R1, 74\% in trial 8 stretch R2) the shared control accuracy is much lower $(67 \%$ and $53 \%$, respec-

Table 3

Inter-trial differences in performance: subject 1, session 4

\begin{tabular}{lllll}
\hline Trial & Stretch & BCI Acc. & Shared control Acc. & Wheelchair behavior \\
\hline 2 & R1 & $92 \%$ & $67 \%$ & Right \\
& R2 & $48 \%$ & $68 \%$ & Right \\
8 & R1 & $65 \%$ & $76 \%$ & Right \\
& R2 & $74 \%$ & $53 \%$ & Right \\
\hline
\end{tabular}

tively). The opposite happens when the BCI accuracy is not that good (trial 2 stretch R2 and trial 8 stretch R1). The implication for the subjects is that they need to learn a model of the shared control system (and its interaction with the $\mathrm{BCI}$ ) to develop successful driving strategies, otherwise their BCI proficiency cannot be fully exploited and, eventually, can hamper the behavior of the wheelchair. But for the subjects to learn that model they need to have a stable performance of the brain-actuated wheelchair. Table 2 shows that, in many cases, the shared control accuracy is rather stable independently of the performance of the BCI (see, in particular, trial 2).

\subsection{Task 2}

Subject 1 reached the final goal in $80 \%$ of the trials. He failed in the last 2 trials, where he was not able to turn Right at the starting point. Making this first Right turn requires a very high $\mathrm{BCI}$ performance because the subject has to rotate the wheelchair by $90^{\circ}$ being almost in the same place (i.e., without entering the corridor it is facing). Indeed, the execution of even a short number of wrong commands in this context makes the shared control system to move the wheelchair Forward. Once the wheelchair is in the corridor, the shared control system makes it very hard to turn back $\left(180^{\circ}\right)$ rapidly and the trial is considered a failure. To illustrate the behavior of the brain-actuated wheelchair in this task, we have included a video clip (see the Supplementary video) which contains the trajectories generated on trials 7 (successful) and 10 (unsuccessful). 


\section{Conclusions}

In this paper we have presented an asynchronous and non-invasive EEG-based BCI prototype for brain-actuated wheelchair driving. The system can be autonomously operated by the user without the need for adaptive algorithms externally tuned by a human operator to minimize the impact of EEG non-stationarities. Our brain-actuated wheelchair has two key components. First, the selection of stable user-specific EEG features that maximize the separability between the patterns generated by executing different mental tasks. Second, the inclusion of a shared control system between the BCI system and the intelligent simulated wheelchair. The reported experiments with two subjects have shown that both were able to reach $90 \%$ (subject 1 ) and $80 \%$ (subject 2) of the goals one day after the calibration of the BCI system, and $100 \%$ (subject 1) and $70 \%$ (subject 2) two months later. It is worth noting that both subjects reached less goals in the first session (one hour after the calibration of the BCI system) and in the third session (two months after the calibration of the BCI system), sessions where the subjects learn or re-learn how to interact with the system and its dynamics. As a consequence, subjects need to cope with the need to generate stable EEG patterns even in the presence of distracting events such as unexpected trajectories of the wheelchair due to the interaction between its intelligence and the context. However, even in these sessions, the subjects showed significant brain-actuated control of the simulated wheelchair: indeed, a random BCI can only reach a mere $1 \%$ of the goals.

In agreement with the results obtained in Vanacker et al. (2007), the analysis over different path stretches shows that the shared control system boosts the BCI performance when it is low, while it may even degrade it when the BCI performance is higher because the user driving strategy is not compatible with the context-based filter. This could explain why subject 1 achieves better performance in task 1 than subject 2 despite the lower LDA classification accuracies on the calibration session (see Table 1). As a consequence, the subject has to learn when these situations occur in order to develop successful driving strategies compatible with the rules of the shared control system. On the other hand, a low BCI accuracy during the driving tasks does not necessarily imply that the BCI is not working correctly. This accuracy is estimated according to the user's stated intent and/or the optimal command for each stretch, while for a proper control of the wheelchair subjects need to make steering corrections and so switch rapidly between mental commands. For this reason we believe that the assessment of an intelligent brain-actuated device cannot simply be based on the BCI performance. As illustrated by the results achieved in task 2, our approach makes it possible for subject 1 to drive along complex paths once he was "free" to concentrate on the task, as he did not need to inform the operator of the mental commands he intended to deliver to the wheelchair.
In this article, we have demonstrated our approach with healthy subjects. However, it is worth noting that our approach should also work for disabled people since it is based on an individual calibration. This calibration procedure, which is common to all users, selects user-specific features that are relevant and stable. In addition, the approach is not based on a fixed set of mental tasks, but subjects can choose those tasks they feel more comfortable with and yield EEG patterns that are more discriminant among themselves.

This discussion brings up a critical issue of a BCI, namely training. Several groups have demonstrated that subjects can learn to control their brain activity through appropriate, but lengthy, training in order to generate fixed EEG patterns that the BCI transforms into external actions (Birbaumer et al., 1999; Wolpaw and McFarland, 2004). In this case the subject is trained over several months to modify the amplitude of their EEG signals following bio-feedback approaches. Contrarily, we follow a mutual learning process to facilitate and accelerate the user's training period. Subjects still need to learn to modulate their EEG, but not all the training burden is on their shoulders - the use of statistical machine learning facilitates the selection of relevant, stable EEG features and the design of optimal classifiers. As shown for the experiments in task 1, subjects can control the wheelchair since the first day with a performance significantly better than a random BCI.

\section{Acknowledgments}

This work was supported in part by the European IST Programme FET Project FP6-003758 (MAIA), by the Swiss National Science Foundation through the National Centre of Research on "Interactive Multimodal Information Management (IM2)", and by FWO Flanders Project G.0317.05. This paper only reflects the author's views, and funding agencies are not liable for any use that may be made of the information contained herein.

\section{Appendix A. Supplementary data}

Supplementary data associated with this article can be found, in the online version, at doi:10.1016/ j.clinph.2008.06.001.

\section{References}

Bayliss JD. Use of the evoked potential P3 component for control in a virtual apartment. IEEE Trans Neural Syst Rehab Eng 2003;11:113-6.

Birbaumer N, Ghanayim N, Hinterberger T, Iversen I, Kotchoubey B, Kübler A, et al. A spelling device for the paralysed. Nature 1999;398:297-8.

Buttfield A, Ferrez PW, Millán RJ del. Towards a robust BCI: error potentials and online learning. IEEE Trans Neural Sys Rehab Eng 2006;14:164-8.

Carmena JM, Lebedev MA, Crist RE, O'Doherty JE, Santucci DM, Dimitrov DF, et al. Learning to control a brain-machine interface for reaching and grasping by primates. PLoS Biol 2003;1:193-208.

Duda RO, Hart PE, Stork DG. Pattern classification. 2nd ed. New York: John Wiley and Sons; 2001. 
Galán F, Ferrez PW, Oliva F, Guárdia J, Millán J del R. Feature extraction for multi-class BCI using canonical variates analysis. In Proc. 2007 IEEE Int. symposium on intelligent signal processing (WISP) 2007. Available at: http://ieeexplore.ieee.org.

Krzanowski WJ. Principles of multivariate analysis. Oxford: Oxford University Press; 1988.

Lew E, Nuttin M, Ferrez PW, Degeest A, Buttfield A, Vanacker G et al. Noninvasive brain-computer interface for mental control of a simulated wheelchair. In Proc. 3rd Int. Brain-Computer Interface Workshop \& Training Course 2006.

Millán J del R. Adaptive brain interfaces. Comm. of the ACM 2003;46:75-80.

Millán J del R, Renkens F, Mouriño J, Gerstner W. Noninvasive brainactuated control of a mobile robot by human EEG. IEEE Trans. Biomed. Eng. 2004;51:1026-33.

Millán J del R, Buttfield A, Vidaurre C, Krauledat M, Schögl A, Shenoy $\mathrm{P}$, et al. Adaptation in brain-computer interfaces. In: Dornhege $\mathrm{G}$, Millán J del R, Hinterberger T, McFarland D, Müller K-R, editors. Towards brain-computer interfacing. Cambridge: MIT Press; 2007. p. $303-25$.
Nicolelis MAL, Chapin JK. Controling robots with the mind. Sci. Am. 2002;287:46-53.

Obermaier B, Müller GR, Pfurtscheller G. Virtual keyboard controlled by spontaneous EEG activity. IEEE Trans. Neural Sys. Rehab. Eng. 2003;11:422-6.

Philips J, Millán J del R, Vanacker G, Lew E, Galán F, Ferrez PW et al. Adaptive shared control of a brain-actuated simulated wheelchair. In: Proc. 10th int. conf. rehabilitation robotics 2007. Available at: http:// ieeexplore.ieee.org).

Shenoy P, Krauledat M, Blankertz B, Rao RPN, Müller K-R. Towards adaptive classification for BCI. J. Neural. Eng. 2006;3:13-23.

Vanacker G, Millán J del R, Lew E, Ferrez PW, Galán F, Philips J, Van Brussel H, Nuttin M. Context-based filtering for assisted brainactuated wheelchair driving. Comput. Intell. Neurosci; 2007, article ID 25130.

Vidaurre C, Schlogl A, Cabeza R, Scherer R, Pfurtscheller G. A fully online adaptive BCI. IEEE Trans Biomed Eng 2006;53:1214-9.

Wolpaw JR, McFarland DJ. Control of a two-dimensional movement signal by a noninvasive brain-computer interface in humans. Proc Natl Acad Sci USA 2004;101:17849-54. 